\title{
Berechnung von mittleren Schwingungsamplituden nach der Methode der charakteristischen Schwingungen von Müller
}

\author{
ENRIQUE J. BARAN \\ Cátedra de Química Inorgánica, Facultad de Ciencias Exactas, \\ Universidad Nacional de La Plata, La Plata, Argentinien \\ (Z. Naturforsch. 25 a, 1292-1295 [1970] ; eingegangen am 2. Mai 1970)
}

\begin{abstract}
It is shown for many molecules and ions that the „Methode der charakteristischen Schwingungen“ of Müller is usefull for the calculation of mean amplitudes of vibrations if the vibrations are rather characteristic.
\end{abstract}

In den letzten Jahren wurden sehr häufig mittlere Schwingungsamplituden aus spektroskopischen Daten berechnet. Da diese Rechnungen in vielen Fällen nicht einfach sind, wurden dabei oft fehlerhafte Ergebnisse erzielt.

Meistens wurden mittlere Schwingungsamplituden aus der Säkulargleichung:

$$
\left|\sum \boldsymbol{G}^{-1}-\boldsymbol{\Delta} \boldsymbol{E}\right|=0
$$

berechnet ${ }^{1}$. Es wurde bereits erwähnt, daß es sinnvoller ist, die $\sum$-Matrix aus den Eigenvektoren nach:

$$
\sum=\boldsymbol{L} \boldsymbol{\Delta} \boldsymbol{L}^{\prime}
$$

zu berechnen ${ }^{2,3}$. In den Gln. (1) und (2) bedeutet $\sum$ die symmetrisierte mittlere-quadratische Amplitudenmatrix mit den Elementen $\sum_{i j}=\left\langle S_{i} S_{j}\right\rangle$, $S_{i}$ und $S_{j}$ sind Symmetriekoordinaten, $\mathbf{G}^{-1}$ ist die Matrix der kinetischen Energie, $\boldsymbol{E}$ die Einheitsmatrix, $\Delta$ eine Diagonalmatrix mit den Elementen:

$$
\Delta_{i}=\frac{h}{8 \pi^{2} v_{i}} \cdot \operatorname{coth} \frac{h v_{i}}{2 k T}
$$

und $\mathbf{L}$, die Eigenvektormatrix, die die Transformationsmatrix zwischen inneren Symmetriekoordinaten $\boldsymbol{S}$ und Normalkoordinaten $\boldsymbol{Q}$ gmäß $\boldsymbol{S}=\boldsymbol{L} \boldsymbol{Q}$ darstellt.

Bekanntlich gilt zwischen der inversen Matrix der kinetischen Energie $\boldsymbol{G}$ und der $\mathbf{L}$-Matrix die Beziehung

$$
\mathbf{G}=\mathbf{L} \mathbf{L}^{\prime}
$$

Es wurde festgestellt, daß man in vielen Fällen die Näherung $L_{i j}=0$ für $i<j$ einführen kann ${ }^{2,4,5}$, was

Reprints request to Dr. E. J. BARAN, Facultad de Ciencias Exactas, Universidad Nacional de La Plata, Calle 47 Esq. 115, La Plata, Argentina.

1 S. J. Cyvin, Mean Square Amplitudes and Molecular Vibrations, Elsevier, Amsterdam 1968.

2 A. Müller, Z. Phys. Chem. Leipzig 238, 116 [1968]. zu erheblicher Vereinfachung des Rechenproblems führt. Die Berechnung der $\sum$-Matrix kann allgemein nach Gl. (2) erfolgen; die L-Matrix wird nach Gl. (3) mit der oben eingeführten Näherung ermittelt ${ }^{4,5}$.

MüLlER und Mitarb. ${ }^{6}$ haben vor kurzem eine Näherungsmethode entwickelt, mit der es ohne Lösung eines inversen Eigenwertproblems möglich ist, mittlere Schwingungsamplituden im Falle des Vorliegens charakteristischer Frequenzen näherungsweise zu berechnen. Bei dieser Methode wird ein gegebenes kompliziertes Molekül in Bruchstücke von je drei Atome des Typs $\mathrm{XY}_{2}$ bzw. XYZ zerlegt, und die Schwingungsfrequenzen dieser „Pseudo-Moleküle“ abgeschätzt. Mit diesen Werten lassen sich mittlere Schwingungsamplituden aus den $\sum$-Elementen für $n=2$ (XY ${ }_{2}$-Bruchstücke) und $n=3$ (XYZ-Bruchstücke), aus den einfachen bereits angegebenen Formeln ${ }^{6}$ berechnen.

In ihrer Arbeit haben Müller und Mitarb. ${ }^{6}$ diese Methode an einigen Beispielen erläutert und gezeigt, daß die so erhaltenen Werte gut mit denjenigen, die mit einer vollständigeren Methode erhalten wurden, übereinstimmen. Es ist der Zweck dieser Arbeit, mittlere Schwingungsamplituden für eine größere Reihe von Molekülen nach der beschriebenen Näherung zu berechnen, um somit die Gültigkeit derselben an weiteren Beispielen zu prüfen und zu bestätigen.

Aus Tab. 1 sind die mittleren Schwingungsamplituden für eine Reihe von Molekülen und Ionen des Typs $Z Z X Y_{3}\left(C_{3 v}\right)$ zu entnehmen und aus Tab. 2 die

3 A. Müller, B. Krebs, A. Fadini, O. Glemser, S. J. Cyvin, J. Brunvoll, B. N. Cyvin, I. Elvebredd, G. Hagen u. B. VIZI, Z. Naturforsch. 23 a, 1656 [1968].

4 C. J. Peacock u. A. Müller, J. Mol. Spectr. 26, 454 [1968].

5 C. J. Peacock, U. Heidborn u. A. Müller, J. Mol. Spectr. 30, 338 [1969].

6 A. Müller, C. J. Peacock, H. Schulze u. U. Heidborn, J. Mol. Struct. 3, 252 [1969]. 


\begin{tabular}{|c|c|c|c|c|c|c|c|c|c|c|c|}
\hline $\mathrm{ZXY}_{3}$ & $T\left({ }^{\circ} \mathrm{K}\right)$ & $u(\mathrm{XY})$ & $u(\mathrm{XZ})$ & $u(\mathrm{Y} \ldots \mathrm{Y})$ & $u(\mathrm{Y} \ldots \mathrm{Z})$ & $\mathrm{ZXY}_{3}$ & $T\left({ }^{\circ} \mathrm{K}\right)$ & $u(\mathrm{XY})$ & $u(\mathrm{XY})$ & $u(\mathbf{Y} \ldots \mathbf{Y})$ & $u(\mathrm{Y} \ldots \mathrm{Z})$ \\
\hline $\mathrm{OPCl}_{3}$ & $\begin{array}{r}298,2 \\
0\end{array}$ & $\begin{array}{ll}\text { a } & 0,0464 \\
\text { b } & 0,0470 \\
\text { c } & 0,0472 \\
\text { a } & 0,0428 \\
\text { b } & 0,0435 \\
\text { c } & 0,0437\end{array}$ & $\begin{array}{c}0,0354 \\
- \\
0,0359 \\
0,0353 \\
\overline{0} \\
0,0357\end{array}$ & $\begin{array}{c}0,085 \\
0,079 \\
- \\
0,060 \\
0,056 \\
-\end{array}$ & $\begin{array}{c}0,068 \\
-\overline{0} \\
0,069 \\
0,058 \\
\overline{-} \\
0,059\end{array}$ & $\mathrm{BrGeH}_{3}$ & 298,2 & $\begin{array}{cc}\text { a } & 0,0894 \\
\text { b } & 0,0894 \\
\text { c } & 0,0894 \\
\text { a } & 0,0894 \\
\text { b } & 0,0894 \\
\text { c } & 0,0894\end{array}$ & $\begin{array}{c}0,0477 \\
- \\
0,0477 \\
0,0379 \\
\overline{0} \\
0,0379\end{array}$ & $\begin{array}{c}0,156 \\
0,155 \\
- \\
0,155 \\
0,154 \\
-\end{array}$ & $\begin{array}{c}0,134 \\
- \\
0, \overline{145} \\
0,128 \\
\overline{-} \\
0,137\end{array}$ \\
\hline $\mathrm{OPBr}_{3}$ & $\begin{array}{r}298,2 \\
0\end{array}$ & $\begin{array}{ll}\text { a } & 0,0464 \\
\text { b } & 0,0507 \\
\text { c } & 0,0490 \\
\text { a } & 0,0407 \\
\text { b } & 0,0435 \\
\text { c } & 0,0427\end{array}$ & $\begin{array}{c}0,0359 \\
- \\
0,0371 \\
0,0357 \\
- \\
0,0366\end{array}$ & $\begin{array}{c}0,090 \\
0,084 \\
-\overline{0} \\
0,051 \\
0,050 \\
-\end{array}$ & $\begin{array}{c}0,077 \\
- \\
0,078 \\
0,059 \\
- \\
0,061\end{array}$ & $\mathrm{IGeH}_{3}$ & 298,2 & $\begin{array}{cc}\text { a } & 0,0895 \\
\text { b } & 0,0895 \\
\text { c } & 0,0895 \\
\text { a } & 0,0895 \\
\text { b } & 0,0895 \\
\text { c } & 0,0895\end{array}$ & $\begin{array}{c}0,0517 \\
- \\
0,0524 \\
0,0380 \\
- \\
0,0384\end{array}$ & $\begin{array}{c}0,157 \\
0,156 \\
- \\
0,155 \\
0,155 \\
-\end{array}$ & $\begin{array}{c}0,143 \\
\overline{-} \\
0, \overline{151} \\
0,134 \\
\overline{-} \\
0,141\end{array}$ \\
\hline $\mathrm{HPO}_{3}^{2-}$ & $\begin{array}{r}298,2 \\
0\end{array}$ & $\begin{array}{ll}\text { a } & 0,0395 \\
\text { b } & 0,0395 \\
\text { c } & 0,0392 \\
\text { a } & 0,0392 \\
\text { b } & 0,0392 \\
\text { c } & 0,0389\end{array}$ & $\begin{array}{c}0,0862 \\
- \\
0,0863 \\
0,0862 \\
- \\
0,0863\end{array}$ & $\begin{array}{c}0,063 \\
0,060 \\
- \\
0,058 \\
0,056 \\
-\end{array}$ & $\begin{array}{c}0,109 \\
- \\
0, \overline{108} \\
0,109 \\
-\overline{107}\end{array}$ & $\mathrm{HGeCl}_{3}$ & 298,2 & $\begin{array}{ll}\text { a } & 0,0461 \\
\text { b } & 0,0465 \\
\text { c } & 0,0462 \\
\text { a } & 0,0405 \\
\text { b } & 0,0408 \\
\text { c } & 0,0406\end{array}$ & $\begin{array}{c}0,0886 \\
- \\
0,0887 \\
0,0886 \\
\overline{0} \\
0,0887\end{array}$ & $\begin{array}{c}0,102 \\
0,098 \\
- \\
0,066 \\
0,064 \\
-\end{array}$ & $\begin{array}{c}0,137 \\
\overline{-} \\
0, \overline{131} \\
0, \overline{130} \\
\overline{-} \\
0, \overline{127}\end{array}$ \\
\hline $\mathrm{HOPO}_{3}^{2-}$ & $\begin{array}{r}298,2 \\
0\end{array}$ & $\begin{array}{ll}\text { a } & 0,0398 \\
\text { b } & 0,0397 \\
\text { c } & 0,0394 \\
\text { a } & 0,0394 \\
\text { b } & 0,0394 \\
\text { c } & 0,0391\end{array}$ & $\begin{array}{c}0,0415 \\
- \\
0,0420 \\
0,0409 \\
- \\
0,0415\end{array}$ & $\begin{array}{c}0,059 \\
0,059 \\
- \\
0,056 \\
0,056 \\
-\end{array}$ & $\begin{array}{c}0,067 \\
- \\
0,069 \\
0,060 \\
- \\
0,061\end{array}$ & $\mathrm{HGeBr}_{3}$ & 298,2 & $\begin{array}{ll}\text { a } & 0,0476 \\
\text { b } & 0,0488 \\
\text { c } & 0,0478 \\
\text { a } & 0,0376 \\
\text { b } & 0,0384 \\
\text { c } & 0,0380\end{array}$ & $\begin{array}{c}0,0895 \\
- \\
0,0899 \\
0,0895 \\
- \\
0,0897\end{array}$ & $\begin{array}{c}0,109 \\
0,104 \\
- \\
0,056 \\
0,055 \\
-\end{array}$ & $\begin{array}{c}0,142 \\
\overline{-} \\
0, \overline{133} \\
0,132 \\
-\overline{129}\end{array}$ \\
\hline $\mathrm{OVF}_{3}$ & 298,2 & $\begin{array}{ll}\text { a } & 0,0404 \\
\text { b } & 0,0409 \\
\text { c } & 0,0410 \\
\text { a } & 0,0394 \\
\text { b } & 0,0399 \\
\text { c } & 0,0399\end{array}$ & $\begin{array}{c}0,0366 \\
- \\
0,0365 \\
0,0364 \\
- \\
0,0362\end{array}$ & $\begin{array}{c}0,098 \\
0,092 \\
- \\
0,072 \\
0,070 \\
-\end{array}$ & $\begin{array}{c}0,079 \\
- \\
0,076 \\
0,065 \\
\overline{-} \\
0,064\end{array}$ & $\mathrm{ClGeBr}_{3}$ & 298,2 & $\begin{array}{ll}\text { a } & 0,0477 \\
\text { b } & 0,0498 \\
\text { c } & 0,0481 \\
\text { a } & 0,0376 \\
\text { b } & 0,0387 \\
\text { c } & 0,0381\end{array}$ & $\begin{array}{c}0,0472 \\
- \\
0,0465 \\
0,0411 \\
- \\
0,0410\end{array}$ & $\begin{array}{c}0,111 \\
0,109 \\
- \\
0,057 \\
0,057 \\
-\end{array}$ & $\begin{array}{c}0,099 \\
- \\
0, \overline{098} \\
0,060 \\
\overline{-} \\
0, \overline{059}\end{array}$ \\
\hline $\mathrm{OVCl}_{3}$ & 298,2 & $\begin{array}{ll}\text { a } & 0,0459 \\
\text { b } & 0,0470 \\
\text { c } & 0,0469 \\
\text { a } & 0,0411 \\
\text { b } & 0,0420 \\
\text { c } & 0,0420\end{array}$ & $\begin{array}{c}0,0369 \\
- \\
0,0372 \\
0,0366 \\
- \\
0,0363\end{array}$ & $\begin{array}{c}0,112 \\
0,108 \\
- \\
0,068 \\
0,067 \\
-\end{array}$ & $\begin{array}{c}0,090 \\
- \\
0,085 \\
0,067 \\
- \\
0,065\end{array}$ & $\mathrm{FGeH}_{3}$ & 298,2 & $\begin{array}{cc}\text { a } & 0,0893 \\
\text { b } & 0,0893 \\
\text { c } & 0,0893 \\
\text { a } & 0,0893 \\
\text { b } & 0,0893 \\
\text { c } & 0,0893\end{array}$ & $\begin{array}{c}0,0415 \\
\overline{-} \\
0,0418 \\
0,0401 \\
\overline{0} \\
0,0403\end{array}$ & $\begin{array}{c}0,156 \\
0,154 \\
- \\
0,154 \\
0,153 \\
-\end{array}$ & $\begin{array}{c}0,125 \\
\overline{-} \\
0, \overline{131} \\
0,122 \\
-\overline{127}\end{array}$ \\
\hline $\mathrm{OVBr}_{3}$ & 298,2 & $\begin{array}{ll}\text { a } & 0,0465 \\
\text { b } & 0,0498 \\
\text { c } & 0,0491 \\
\text { a } & 0,0384 \\
\text { b } & 0,0401 \\
\text { c } & 0,0402\end{array}$ & $\begin{array}{c}0,0371 \\
- \\
0,0381 \\
0,0368 \\
- \\
0,0374\end{array}$ & $\begin{array}{c}0,119 \\
0,112 \\
- \\
0,058 \\
0,057 \\
-\end{array}$ & $\begin{array}{c}0,097 \\
- \\
0,092 \\
0,067 \\
- \\
0,066\end{array}$ & $\mathrm{ClGeH}_{3}$ & 298,2 & $\begin{array}{cc}\text { a } & 0,0893 \\
\text { b } & 0,0893 \\
\text { c } & 0,0892 \\
\text { a } & 0,0893 \\
\text { b } & 0,0893 \\
\text { c } & 0,0893\end{array}$ & $\begin{array}{c}0,0464 \\
- \\
0,0467 \\
0,0407 \\
\overline{0} \\
0,0409\end{array}$ & $\begin{array}{c}0,156 \\
0,155 \\
- \\
0,154 \\
0,153 \\
-\end{array}$ & $\begin{array}{c}0,134 \\
-\overline{141} \\
0,141 \\
0,128 \\
\overline{-} \\
0, \overline{134}\end{array}$ \\
\hline $\mathrm{ClReO}_{3}$ & 298,2 & $\begin{array}{cc}\text { a } & 0,0346 \\
\text { b } & 0,0345 \\
\text { c } & 0,0346 \\
\text { a } & 0,0343 \\
\text { b } & 0,0342 \\
\text { c } & 0,0342\end{array}$ & $\begin{array}{c}0,0550 \\
- \\
0,0563 \\
0,0432 \\
- \\
0,0439\end{array}$ & $\begin{array}{c}0,068 \\
0,063 \\
- \\
0,060 \\
0,057 \\
-\end{array}$ & $\begin{array}{c}0,091 \\
- \\
0,099 \\
0,067 \\
\overline{-} \\
0,071\end{array}$ & $\mathrm{ISiBr}_{3}$ & 298.2 & $\begin{array}{ll}\text { a } & 0,0463 \\
\text { b } & 0,0342 \\
\text { c } & 0,0339 \\
\text { a } & 0,0414 \\
\text { b } & 0,0308 \\
\text { c } & 0,0306\end{array}$ & $\begin{array}{c}0,0648 \\
\overline{-} \\
0,0565 \\
0,0487 \\
\overline{0} \\
0,0399\end{array}$ & $\begin{array}{c}0,118 \\
0,088 \\
- \\
0,057 \\
0,050 \\
-\end{array}$ & $\begin{array}{c}0,087 \\
\overline{-} \\
0, \overline{126} \\
0,049 \\
\overline{-} \\
0, \overline{057}\end{array}$ \\
\hline $\mathrm{BrReO}_{3}$ & 298,2 & $\begin{array}{cc}\text { a } & 0,0346 \\
\text { b } & 0,0345 \\
\text { c } & 0,0345 \\
\text { a } & 0,0343 \\
\text { b } & 0,0342 \\
\text { c } & 0,0342\end{array}$ & $\begin{array}{c}0,0571 \\
- \\
0,0593 \\
0,0382 \\
- \\
0,0393\end{array}$ & $\begin{array}{c}0,070 \\
0,069 \\
- \\
0,061 \\
0,060 \\
-\end{array}$ & $\begin{array}{c}0,099 \\
- \\
0, \overline{108} \\
0,066 \\
- \\
0, \overline{070}\end{array}$ & $\mathrm{ClSiH}_{3}$ & 298,2 & $\begin{array}{cc}\text { a } & 0,0888 \\
\text { b } & 0,0888 \\
\text { c } & 0,0895 \\
\text { a } & 0,0888 \\
\text { b } & 0,0888 \\
\text { c } & 0,0895\end{array}$ & $\begin{array}{l}0,0461 \\
- \\
0, \overline{0474} \\
0,0431 \\
\overline{-} \\
0,0441\end{array}$ & $\begin{array}{c}0,151 \\
0,150 \\
- \\
0,150 \\
0,149 \\
-\end{array}$ & $\begin{array}{c}0,128 \\
\overline{-} \\
0, \overline{134} \\
0,125 \\
-\overline{130}\end{array}$ \\
\hline $\mathrm{SReO}_{3}$ & 298,2 & $\begin{array}{cc}\text { a } & 0,0360 \\
\text { b } & 0,0358 \\
\text { c } & 0,0360 \\
\text { a } & 0,0357 \\
\text { b } & 0,0354 \\
\text { c } & 0,0355\end{array}$ & $\begin{array}{c}0,0384 \\
- \\
0,0382 \\
0,0351 \\
\overline{-} \\
0,0350\end{array}$ & $\begin{array}{c}0,074 \\
0,073 \\
- \\
0,063 \\
0,062 \\
-\end{array}$ & $\begin{array}{c}0,077 \\
- \\
0,081 \\
0,062 \\
- \\
0,063\end{array}$ & $\mathrm{BrSiH}_{3}$ & 298,2 & $\begin{array}{cc}\text { a } & 0,0888 \\
\text { b } & 0,0888 \\
\text { c } & 0,0888 \\
\text { a } & 0,0888 \\
\text { b } & 0,0888 \\
\text { c } & 0,0888\end{array}$ & $\begin{array}{c}0,0476 \\
\overline{-} \\
0,0492 \\
0,0422 \\
\overline{-} \\
0,0434\end{array}$ & $\begin{array}{c}0,151 \\
0,150 \\
- \\
0,150 \\
0,149 \\
-\end{array}$ & $\begin{array}{c}0,132 \\
\overline{-} \\
0, \overline{138} \\
0,127 \\
\overline{-} \\
0,132\end{array}$ \\
\hline
\end{tabular}




\begin{tabular}{|c|c|c|c|c|c|c|c|c|c|c|c|}
\hline $\mathrm{ZXY}_{3}$ & $T\left({ }^{\circ} \mathrm{K}\right)$ & $u(\mathrm{XY})$ & $u(\mathrm{XZ})$ & $u(\mathrm{Y} \ldots \mathrm{Y})$ & $u(\mathrm{Y} \ldots \mathrm{Z})$ & $\mathrm{ZXY}_{3}$ & $T\left({ }^{\circ} \mathrm{K}\right)$ & $u(\mathrm{XY})$ & $u(\mathrm{XZ})$ & $u(\mathrm{Y} \ldots \mathrm{Y})$ & $u(\mathrm{Y} \ldots \mathrm{Z})$ \\
\hline $\mathrm{ISiH}_{3}$ & 298,2 & $\begin{array}{ll}\text { a } & 0,0887 \\
\text { b } & 0,0888 \\
\text { c } & 0,0887 \\
\text { a } & 0,0887 \\
\text { b } & 0,0888 \\
\text { c } & 0,0887\end{array}$ & $\begin{array}{c}0,0516 \\
- \\
0,0536 \\
0,0435 \\
\overline{-} \\
0,0449\end{array}$ & $\begin{array}{c}0,152 \\
0.151 \\
- \\
0,151 \\
0,150 \\
-\end{array}$ & $\begin{array}{c}0,139 \\
-\overline{14} \\
0, \overline{146} \\
0,132 \\
\overline{-} \\
0, \overline{138}\end{array}$ & $\mathrm{SSO}_{3}^{2-}$ & $\begin{array}{c}298,2 \\
0\end{array}$ & $\begin{array}{ll}\text { a } & 0,0390 \\
\text { b } & 0,0389 \\
\text { c } & 0,0386 \\
\text { a } & 0,0387 \\
\text { b } & 0,0386 \\
\text { c } & 0,0384\end{array}$ & $\begin{array}{c}0,0467 \\
- \\
0,0541 \\
0,0429 \\
- \\
0,0482\end{array}$ & $\begin{array}{c}0,059 \\
0,056 \\
- \\
0,056 \\
0,053 \\
-\end{array}$ & $\begin{array}{c}0,069 \\
-\overline{73} \\
0,073 \\
0,059 \\
-\overline{062}\end{array}$ \\
\hline $\mathrm{HSiF}_{3}$ & $\begin{array}{r}298,2 \\
\qquad\end{array}$ & $\begin{array}{ll}\text { a } & 0,0402 \\
\text { b } & 0,0404 \\
\text { c } & 0,0399 \\
\text { a } & 0,0397 \\
\text { b } & 0,0399 \\
\text { c } & 0,0395\end{array}$ & $\begin{array}{c}0,0865 \\
-\overline{0} \\
0,0866 \\
0,0865 \\
\overline{-} \\
0,0866\end{array}$ & $\begin{array}{c}0,075 \\
0,071 \\
- \\
0,063 \\
0,061 \\
-\end{array}$ & $\begin{array}{c}0,117 \\
\overline{-} \\
0,115 \\
0,115 \\
\overline{-} \\
0, \overline{113}\end{array}$ & $\mathrm{HSO}_{3}^{2-}$ & $\begin{array}{r}298,2 \\
0\end{array}$ & $\begin{array}{ll}\text { a } & 0,0375 \\
\text { b } & 0,0376 \\
\text { c } & 0,0372 \\
\text { a } & 0,0373 \\
\text { b } & 0,0375 \\
\text { c } & 0,0371\end{array}$ & $\begin{array}{c}0,0812 \\
- \\
0,0813 \\
0,0812 \\
- \\
0,0813\end{array}$ & $\begin{array}{c}0,059 \\
0,057 \\
- \\
0,055 \\
0,054 \\
-\end{array}$ & $\begin{array}{l}0,104 \\
- \\
0, \overline{102} \\
0,103 \\
-\overline{102}\end{array}$ \\
\hline $\mathrm{HSiCl}_{3}$ & $\begin{array}{r}298,2 \\
0\end{array}$ & $\begin{array}{ll}\text { a } & 0,0464 \\
\text { b } & 0,0480 \\
\text { c } & 0,0472 \\
\text { a } & 0,0432 \\
\text { b } & 0,0445 \\
\text { c } & 0,0441\end{array}$ & $\begin{array}{c}0,0876 \\
- \\
0,0897 \\
0,0876 \\
- \\
0,0879\end{array}$ & $\begin{array}{c}0,090 \\
0,086 \\
- \\
0,062 \\
0,061 \\
-\end{array}$ & $\begin{array}{c}0,129 \\
-\overline{122} \\
0,122 \\
0,125 \\
-\overline{120}\end{array}$ & $\mathrm{FSO}_{3}^{2-}$ & 298,2 & $\begin{array}{ll}\text { a } & 0,0364 \\
\text { b } & 0,0366 \\
\text { c } & 0,0361 \\
\text { a } & 0,0363 \\
\text { b } & 0,0365 \\
\text { c } & 0,0360\end{array}$ & $\begin{array}{c}0,0426 \\
- \\
0, \overline{0433} \\
0,0416 \\
- \\
0, \overline{0423}\end{array}$ & $\begin{array}{c}0,055 \\
0,055 \\
- \\
0,053 \\
0,053 \\
-\end{array}$ & $\begin{array}{c}0,064 \\
\overline{-} \\
0,065 \\
0,058 \\
\overline{-} \\
0,059\end{array}$ \\
\hline $\mathrm{HSiBr}_{3}$ & 298,2 & $\begin{array}{ll}\text { a } & 0,0476 \\
\text { b } & 0,0502 \\
\text { c } & 0,0489 \\
\text { a } & 0,0420 \\
\text { b } & 0,0438 \\
\text { c } & 0,0432\end{array}$ & $\begin{array}{c}0,0880 \\
- \\
0,0887 \\
0,0880 \\
- \\
0,0885\end{array}$ & $\begin{array}{c}0,094 \\
0,091 \\
- \\
0,053 \\
0,052 \\
-\end{array}$ & $\begin{array}{c}0,134 \\
- \\
0, \overline{124} \\
0,127 \\
\overline{-} \\
0, \overline{122}\end{array}$ & $\mathrm{HOSO}_{3}^{2-}$ & $\begin{array}{r}298,2 \\
0\end{array}$ & $\begin{array}{ll}\text { a } & 0,0377 \\
\text { b } & 0,0376 \\
\text { c } & 0,0372 \\
\text { a } & 0,0374 \\
\text { b } & 0,0374 \\
\text { c } & 0,0371\end{array}$ & $\begin{array}{c}0,0411 \\
- \\
0,0419 \\
0,0405 \\
- \\
0,0413\end{array}$ & $\begin{array}{c}0,056 \\
0,055 \\
- \\
0,053 \\
0,053 \\
-\end{array}$ & $\begin{array}{c}0,063 \\
-\overline{64} \\
0,064 \\
0,058 \\
\overline{-} \\
0,059\end{array}$ \\
\hline $\mathrm{FSiCl}_{3}$ & 298,2 & $\begin{array}{ll}\text { a } & 0,0416 \\
\text { b } & 0,0453 \\
\text { c } & 0,0406 \\
\text { a } & 0,0395 \\
\text { b } & 0,0422 \\
\text { c } & 0,0392\end{array}$ & $\begin{array}{c}0,0413 \\
- \\
0,0410 \\
0,0407 \\
\overline{0} \\
0,0405\end{array}$ & $\begin{array}{c}0,092 \\
0,094 \\
- \\
0,062 \\
0,064 \\
-\end{array}$ & $\begin{array}{c}0,078 \\
-\overline{0} \\
0,076 \\
0,062 \\
\overline{-} \\
0,061\end{array}$ & $\mathrm{HOSeO}_{3}^{2-}$ & 298,2 & $\begin{array}{ll}\text { a } & 0,0381 \\
\text { b } & 0,0382 \\
\text { c } & 0,0380 \\
\text { a } & 0,0376 \\
\text { b } & 0,0377 \\
\text { c } & 0,0375\end{array}$ & $\begin{array}{c}0,0411 \\
- \\
0,0414 \\
0,0400 \\
- \\
0,0403\end{array}$ & $\begin{array}{c}0,068 \\
0,067 \\
- \\
0,061 \\
0,060 \\
-\end{array}$ & $\begin{array}{l}0,074 \\
\overline{-} \\
0,075 \\
0,064 \\
\overline{-} \\
0,064\end{array}$ \\
\hline $\mathrm{BrSiCl}_{3}$ & 298,2 & $\begin{array}{ll}\text { a } & 0,0460 \\
\text { b } & 0,0457 \\
\text { c } & 0,0453 \\
\text { a } & 0,0429 \\
\text { b } & 0,0430 \\
\text { c } & 0,0427\end{array}$ & $\begin{array}{c}0,0502 \\
- \\
0,0551 \\
0,0433 \\
- \\
0,0465\end{array}$ & $\begin{array}{c}0,106 \\
0,087 \\
- \\
0,066 \\
0,061 \\
-\end{array}$ & $\begin{array}{c}0,077 \\
- \\
0, \overline{109} \\
0,053 \\
-\overline{0} \\
0,063\end{array}$ & $\mathrm{FCrO}_{3}^{-}$ & $\begin{array}{c}298,2 \\
0\end{array}$ & $\begin{array}{ll}\text { a } & 0,0389 \\
\text { b } & 0,0389 \\
\text { c } & 0,0387 \\
\text { a } & 0,0384 \\
\text { b } & 0,0384 \\
\text { c } & 0,0383\end{array}$ & $\begin{array}{c}0,0454 \\
- \\
0,0457 \\
0,0433 \\
- \\
0, \overline{0436}\end{array}$ & $\begin{array}{c}0,071 \\
0,071 \\
- \\
0,063 \\
0,062 \\
-\end{array}$ & $\begin{array}{c}0,086 \\
- \\
0,088 \\
0,069 \\
- \\
0,069\end{array}$ \\
\hline $\mathrm{ClSiBr}_{3}$ & 298,2 & $\begin{array}{ll}\text { a } & 0,0477 \\
\text { b } & 0,0513 \\
\text { c } & 0,0671 \\
\text { a } & 0,0419 \\
\text { b } & 0,0441 \\
\text { c } & 0,0525\end{array}$ & $\begin{array}{c}0,0471 \\
- \\
0,0477 \\
0,0437 \\
\overline{-} \\
0,0440\end{array}$ & $\begin{array}{c}0,100 \\
0,080 \\
- \\
0,054 \\
0,049 \\
-\end{array}$ & $\begin{array}{c}0,084 \\
- \\
0,087 \\
0,056 \\
-\overline{057}\end{array}$ & $\mathrm{ClCrO}_{3}^{-}$ & 298,2 & $\begin{array}{ll}\text { a } & 0,0388 \\
\text { b } & 0,0389 \\
\text { c } & 0,0387 \\
\text { a } & 0,0384 \\
\text { b } & 0,0385 \\
\text { c } & 0,0383\end{array}$ & $\begin{array}{c}0,0485 \\
- \\
0,0481 \\
0,0426 \\
- \\
0,0426\end{array}$ & $\begin{array}{c}0,072 \\
0,072 \\
- \\
0,063 \\
0,063 \\
-\end{array}$ & $\begin{array}{l}0,093 \\
\overline{-} \\
0,099 \\
0,069 \\
\overline{-} \\
0,071\end{array}$ \\
\hline $\mathrm{ISiCl}_{3}$ & $\begin{array}{r}298,2 \\
0\end{array}$ & $\begin{array}{ll}\text { a } & 0,0467 \\
\text { b } & 0,0467 \\
\text { c } & 0,0461 \\
\text { a } & 0,0435 \\
\text { b } & 0,0437 \\
\text { c } & 0,0433\end{array}$ & $\begin{array}{c}0,0510 \\
- \\
0,0567 \\
0,0429 \\
- \\
0,0464\end{array}$ & $\begin{array}{c}0,115 \\
0,109 \\
- \\
0,069 \\
0,068 \\
-\end{array}$ & $\begin{array}{c}0,078 \\
-\overline{115} \\
0,052 \\
0,052 \\
- \\
0,063\end{array}$ & $\mathrm{NOsO}_{3}^{-}$ & $\begin{array}{r}298,2 \\
\\
0\end{array}$ & $\begin{array}{ll}\text { a } & 0,0366 \\
\text { b } & 0,0365 \\
\text { c } & 0,0366 \\
\text { a } & 0,0360 \\
\text { b } & 0,0360 \\
\text { c } & 0,0360\end{array}$ & $\begin{array}{c}0,0359 \\
- \\
0,0358 \\
0,0356 \\
- \\
0,0356\end{array}$ & $\begin{array}{c}0,069 \\
0,069 \\
- \\
0,061 \\
0,061 \\
-\end{array}$ & $\begin{array}{c}0,076 \\
- \\
0,076 \\
0,065 \\
-\overline{065}\end{array}$ \\
\hline
\end{tabular}

Tab. 1. Mittlere Schwingungsamplituden für $\mathrm{ZXY}_{3}\left(\mathrm{C}_{3 \mathrm{v}}\right)-$ Moleküle. Werte in $\AA$, erhalten von: a) vollständige Rechnung ${ }^{3}$; b) $\mathrm{XY}_{2}$-Bruchstück; c) ZXY-Bruchstück.

entsprechenden Werte für Moleküle des Typs $\mathrm{ZXY}_{2}$ $\left(\mathrm{C}_{2 \mathrm{v}}\right)$. Die Rechnungen wurden für zwei Temperaturen $\left(298,2\right.$ und $\left.0{ }^{\circ} \mathrm{K}\right)$ durchgeführt und die erhaltenen Werte jeweils mit den Werten verglichen, die mit vollständiger Rechnung erhalten wurden. Die Schwingungsfrequenzen sind die gleichen wie bei ${ }^{3}$. Zur Mittelung bei entarteten Schwingungen vgl. ${ }^{6}$.
Wie leicht zu ersehen ist, liefern die Näherungsrechnungen brauchbare Werte, vor allem wenn das Zentralatom relativ schwerer ist; aber auch in allen Fällen und auch bei den Amplituden für nicht gebundene Atome $[u(\mathrm{Y} \ldots \mathrm{Y})$ und $u(\mathrm{Y} \ldots \mathrm{Z})]$ ist die Übereinstimmung relativ gut. Die Unterschiede zu den Literaturwerten sind lediglich in wenigen 


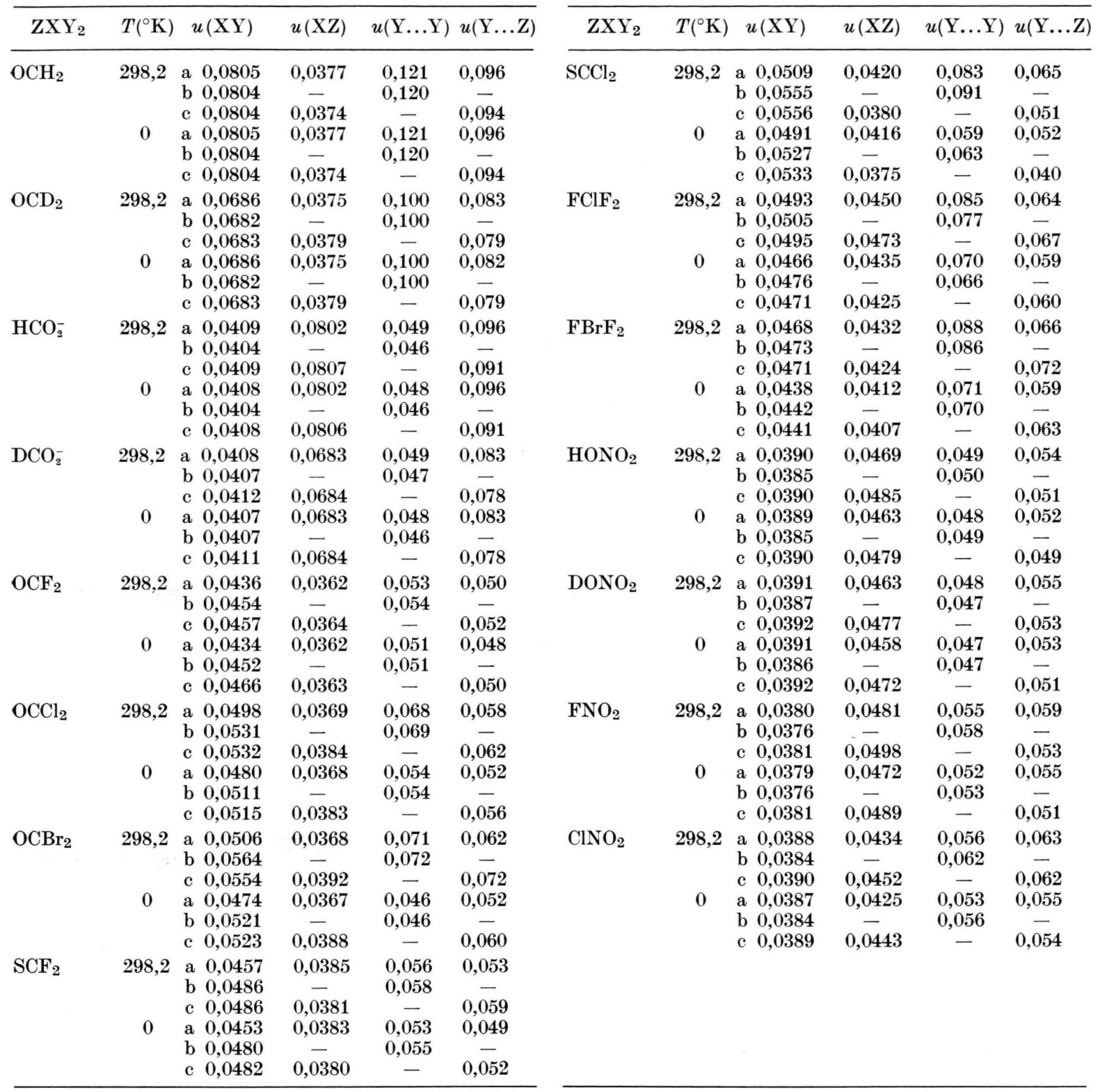

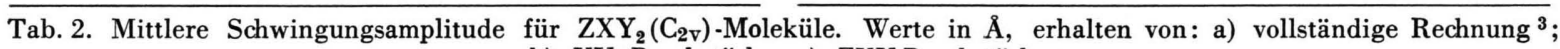
b) $\mathrm{XY}_{2}$-Bruchstück; c) ZXY-Bruchstück.

Fällen größer als 5\%, und zwar dann, wenn die Masse der Außenatome im Verhältnis zur Masse des Zentralatoms groß ist und vor allen Dingen, wenn mehrere schwere Außenatome vorhanden sind (vgl. z. B. $\mathrm{ISiBr}_{3}, \mathrm{OPBr}_{3}, \mathrm{HSiBr}_{3}$ etc.).

Man kann also zusammenfassend und an Hand unserer Rechnungen sagen, daß diese Methode mit geringem Rechenaufwand zu guten Ergebnissen führt und vor allen Dingen für Überschlagsrechnungen außerordentlich brauchbar ist. Die Methode kann man allgemein für ein beliebiges Molekül anwenden, vorausgesetzt, daß die Zuordnung ihrer Schwingungsfrequenzen bekannt ist und charakteristische Schwingungen vorliegen.

Diese Arbeit wurde mit Unterstützung des „Consejo Nacional de Investigaciones Científicas y Técnicas de la República Argentina" und einem Kontrakt der UNLP durchgeführt. 


\title{
Fine Structure of the NH Stretching Band of Pyrrole
}

\author{
JAAKKO K. ElORANTA \\ Laboratory of Molecular Spectroscopy, Department of Physics, University of Oulu, Oulu, Finland \\ (Z. Naturforsch. 25 a, 1296-1299 [1970] ; received 2 May 1970)
}

\begin{abstract}
The rotational fine structure of the fundamental $\mathrm{NH}$ stretching band of pyrrole has been analyzed. The analysis has been based on the symmetric top approximation. The obtained rotational constants $B^{\prime \prime}=0.3032 \mathrm{~cm}^{-1}, B^{\prime}=0.3027 \mathrm{~cm}^{-1}$, and $C=0.1505 \mathrm{~cm}^{-1}$ are in good agreement with the microwave data. Also the accurate band center $v=3530.520 \mathrm{~cm}^{-1}$ has been obtained.
\end{abstract}

According to the microwave structural analysis of the pyrrole molecule ${ }^{1,2}$ the ratio of the two moments of inertia about the two perpendicular axes in the molecular plane is 0.986 . Accordingly, the molecule is very close to an oblate symmetric top and the analysis of the rotational fine structure of the vibrational bands can be performed by applying the theory of the symmetric top molecules. The complete asymmetric top analysis of the infrared spectrum of a heavy molecule like pyrrole would be rather cumbersome and even impossible.

In this paper the fundamental NH stretching band of pyrrole has been analyzed because it fits the region of the high resolution spectrometer constructed in our laboratory. The obtained rotational constants are good approximations to the actual ones and may be compared with the microwave rotational constants. Further, the accurate band center has been obtained which is useful for the research of vibrational anharmonicities in progress at this laboratory.

\section{Experimental}

The high resolution spectrum of the $\mathrm{NH}$ stretching band of pyrrole was recorded by means of a large near-infrared Pfund-type grating spectrometer which has been described elsewhere ${ }^{3}$. The vapor sample was in a 3-meter multiple pass absorption cell in a pressure of about $5 \mathrm{~mm} \mathrm{Hg}$. The relative humidity inside the spectrometer was about 13 per cent causing the superimposed water vapor absorptions which could be identified with the help of the paper of PLYLER and TIDWELL ${ }^{4}$. The spectral slitwidth during the run was $0.14 \mathrm{~cm}^{-1}$. The wavenumbers of the absorption lines were calibrated against the known emission lines of

Sonderdruckanforderungen an Dr. J. EloranTA, Laboratory of Molecular Spectroscopy, Department of Physics, University of Oulu, Kontinkangas, Oulu, Finnland.

1 B. Bak, D. Christensen, L. Hansen, and J. Rastrup-ANDersen, J. Chem. Phys. 24, 720 [1956]. rare gases. The pyrrole used in this work was purchased from $\mathrm{Fl} \mathrm{u} \mathrm{k} \mathrm{a} \mathrm{AG} \mathrm{and} \mathrm{was} \mathrm{of} \mathrm{purissimum} \mathrm{grade} \mathrm{and}$ used without any further purification.

\section{Theoretical}

The rotational term value which is applicable for the non-degenerate vibrational energy states is given by

$$
F(J, K)=B J(J+1)+(C-B) K^{2}-D_{J} J^{2}(J+1)^{2},
$$

when the negligible centrifugal distortion terms dependent on $K$ have been omitted. In this case $B$ is the mean value of the actual rotational constants $A$ and $B$ of a pyrrole molecule.

The vibration of the $\mathrm{NH}$ stretching mode $v_{9}$ of pyrrole is perpendicular to the top axis. According to the selection rules of a perpendicular band, $\Delta J= \pm 1$ and $\Delta K= \pm 1$, the waveumbers of the sub band components can be expressed in terms of $m$ which is $-J$ in the $\mathrm{P}$ branch and $J+1$ in the $\mathbf{R}$ branch of the band,

$$
v=v_{\mathrm{K}}^{\mathrm{sub}}+\left(B^{\prime}+B^{\prime \prime}\right) m+\left(B^{\prime}-B^{\prime \prime}\right) m^{2}-4 D_{\mathrm{J}} m^{3} .
$$

The sub band origins $\nu_{\mathrm{K}}^{\mathrm{sub}}$ are given by

$$
\begin{aligned}
v_{\mathrm{K}}^{\mathrm{sub}}=v_{0} & +\left(C^{\prime}-B^{\prime}\right) \pm 2\left(C^{\prime}-B^{\prime}\right) K \\
& +\left[\left(C^{\prime}-B^{\prime}\right)-\left(C^{\prime \prime}-B^{\prime \prime}\right)\right] K^{2},
\end{aligned}
$$

where $v_{0}$ is the wavenumber of the band center. The + and - signs refer to the positive $(\Delta K=1)$ and negative $(\Delta K=-1)$ sub bands, respectively. The rotational constants of the upper state are denoted by the single prime and those of the lower state by the double prime.

2 L. Nygaard, J. T. Nielsen, J. Kirchheiner, G. Maltesen, J. Rastrup-Andersen, and G. O. Sørensen, J. Mol. Structure 3, 491 [1969].

3 R. Anttila, Ann. Acad. Sci. Fennicae, Ser. A 1967, 254.

${ }^{4}$ E. K. Plyler and E. D. Tidwell, Mem. Soc. Roy. Sci. Liège, 4e ser., 18, 426 [1956]. 


\section{Results}

For the infrared fundamental bands the difference between the rotational constants of the upper and the lower state is small as a rule and thus the last term in the expression of the sub band origin should be small. The spacing of the subsequent sub bands should be $2\left(C^{\prime}-B^{\prime}\right)$, which is about $0.3 \mathrm{~cm}^{-1}$ according to the microwave data. On the other hand the spacing of the $J$ components should be

$$
B^{\prime}+B^{\prime \prime} \sim 0.6 \mathrm{~cm}^{-1} \text {. }
$$

Accordingly, the sub band centers and the $J$ components of different sub bands will be superimposed with the same interval of $0.3 \mathrm{~cm}^{-1}$. One can expect the absorptions to coincide up to rather high values of $J$ and $K$ due to the planarity and the accidental symmetry of the molecule.

On the basis of the foregoing discussion of the structure of the band a theoretical band shape was computed using the Hönl-London intensity formulae ${ }^{5}$. The rotational constants reported by BAK et al. ${ }^{1}$ and the temperature of $300{ }^{\circ} \mathrm{K}$ were used. The difference between the primed rotational con- stants was omitted. Fig. 1 illustrates the sub bands of the lowest five $K$ transitions and the resultant of sub bands up to $K=80$ when every other line has been left out. The small circles and triangles denote the portion of the negative and the positive sub bands, respectively. The cross lines indicate the portion of the $Q$ lines in the pattern.

The calculated spectrum was employed as a reference in the assignment of the spectral lines of the experimental spectrum. Also the experimental spectrum is illustrated in Fig. 1 and one can see the good agreement with the calculated one.

The $B$ constants should be calculated from single sub bands but because of the superposition of the sub bands the individual bands cannot be resolved. Each absorption line is a sum of the sub band lines of different $m$ values and as can be seen in the computed spectrum the portion of the positive sub bands in the $\mathrm{P}$ branch is quite small as is the portion of the negative sub bands in the $R$ branch. The actual asymmetry and on the other hand the non-zero coefficients $B^{\prime}-B^{\prime \prime}$ and $4 D$ may break the coincidence of the lines when $m$ is high. Therefore it is

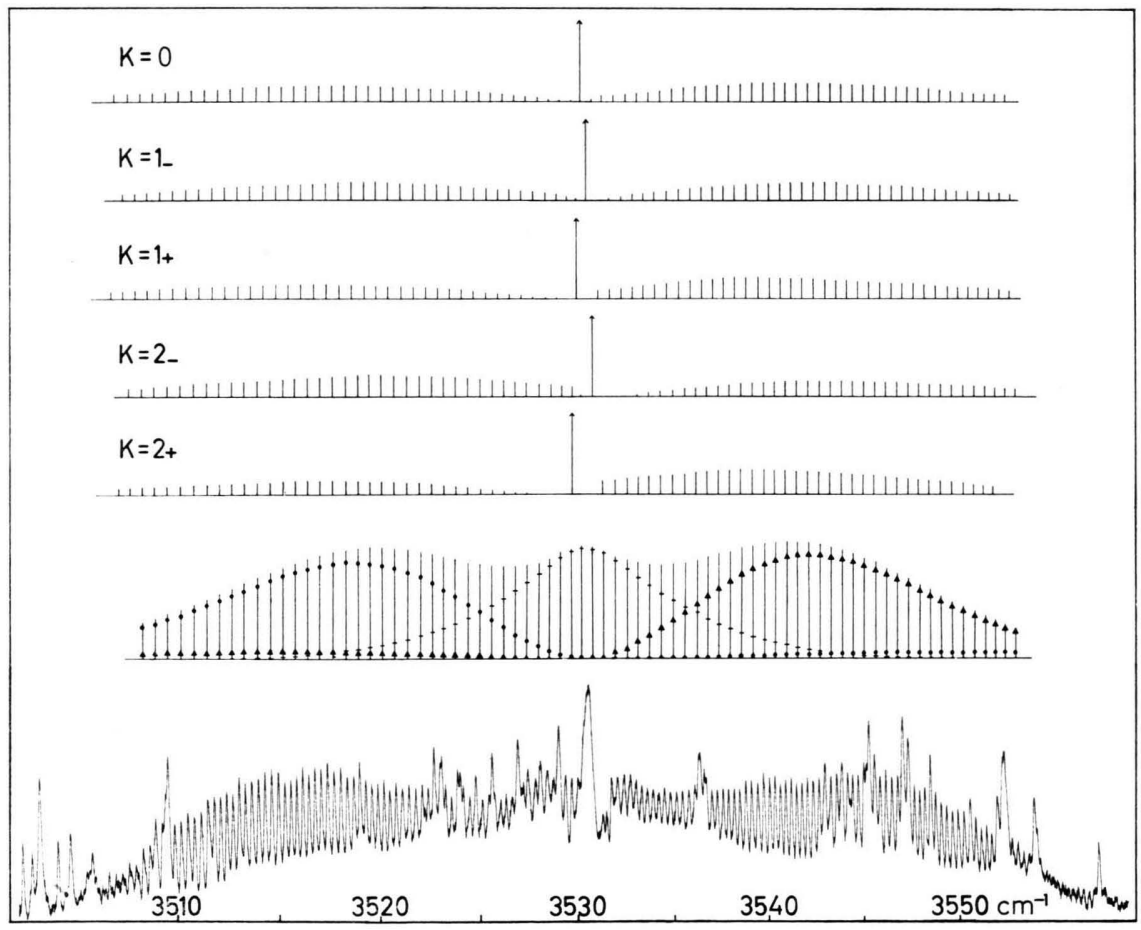

Fig. 1. Fine structure of the $\mathrm{NH}$ stretching band of pyrrole. The upper five spectra are the perpendicular type sub bands of the lowest $K$ values computed with the Hönl-London intensity formulae at $300^{\circ} \mathrm{K}$. The sixth spectrum is the sum of the sub bands. This spectrum is not to the same scale as the upper ones and every other line has been omitted for clarity. The circles and the triangles denote the portion of the negative and the positive sub bands in the line intensity, respectively. The portion of the $Q$ components is indicated by the cross lines. The lowest spectrum is the recorded one. Except for the strong central maximum near $3531 \mathrm{~cm}^{-1}$, which is the parallel part of the hybrid band, all the lines which differ from the calculated ones are due to water vapor absorptions.

\footnotetext{
5 G. Herzberg, Molecular Spectra and Molecular Structure, II. Infrared and Raman Spectra of Polyatomic Molecules,
} D. van Nostrand Co., New York 1964, pp. 424-428. 
not probable that a measured absorption maximum might present the wavenumber of the weak lines.

An attempt was made to compute the rotational constants separately for the positive sub bands from the $\mathrm{R}$ branch absorptions and for the negative sub bands from the $\mathrm{P}$ branch absorptions. The obtained results differed too greatly, probably due to the uneven distribution of the measurable lines over the entire band (cf. Table 1). Therefore, the least square fit over the whole band was used to evaluate the rotational constants. The fits were computed for the sub bands $K=0, K=1_{-}$, and $K=1_{+}$simply by changing the numbering of the absorptions. In order to avoid unrealistic contribution from the unresolved sub band components, $C^{\prime}$ was taken as equal to $C^{\prime \prime}$. The $C$ constant as well as the band origin $v_{0}$ were computed from the obtained sub band origins.

The observed and calculated wavenumbers and their differences are collected in Table 1. The calculated values have been taken from the polynomia] computed for the sub band $K=0$,

$$
\begin{aligned}
v= & 3530.375+0.60594 m-5.10 \times 10^{-4} \mathrm{~m}^{2} \\
& -1 \times 10^{-6} \mathrm{~m}^{3} .
\end{aligned}
$$

The haif integers of $m$ which appear in Table 1 refer to the absorptions which belong to the sub bands with odd $K$ values. The average difference between the measured and calculated wavenumbers is 0.014 $\mathrm{cm}^{-1}$.

The calculated rotational constants and the wavenumber of the band center are presented in Table 2 together with the microwave data. The coefficient of the third power term in the polynomial fits of various sub bands varied so much that the $D_{J}$ constant

\begin{tabular}{|c|c|c|}
\hline \multicolumn{2}{|r|}{ This work } & NYGAaRD et al. \\
\hline$v_{0}$ & $=3530.520$ & \\
\hline$B^{\prime \prime}$ & $=0.3032$ & $A=0.3046$ \\
\hline$B^{\prime}$ & 0.3027 & $B=0.3003$ \\
\hline$C$ & 0.1505 & $C=0.1512$ \\
\hline
\end{tabular}
could not be evaluated reliably.

Table 2.

Molecular constants of pyrrole. All the values are in $\mathrm{cm}^{-1}$

\section{Discussion}

The rotational constants obtained agree well with the microwave data ${ }^{1,2}$. The differences are within

\begin{tabular}{|c|c|c|c|c|c|c|c|}
\hline$m$ & $\begin{array}{l}v_{\mathrm{obs}} \\
\mathrm{cm}^{-1}\end{array}$ & $\begin{array}{l}v_{\text {calc }} \\
\mathrm{cm}^{-1}\end{array}$ & $\begin{array}{c}v_{\text {obs. }}-v_{\text {calc. }} \\
\mathrm{cm}^{-1}\end{array}$ & $m$ & $\begin{array}{l}v_{\text {obs. }} \\
\mathrm{cm}^{-1}\end{array}$ & $\begin{array}{l}v_{\text {calc }} \\
\mathrm{cm}^{-1}\end{array}$ & $\begin{array}{c}v_{\text {obs. }}-v_{\text {calc }} \\
\mathrm{cm}^{-1}\end{array}$ \\
\hline-30 & 3511.747 & 3511.766 & -0.019 & -15.5 & 3520.877 & 3520.865 & 0.012 \\
\hline-29.5 & 12.063 & 12.082 & -0.019 & -15 & 21.173 & 21.175 & -0.002 \\
\hline-29 & 12.374 & 12.399 & -0.025 & -14.5 & 21.472 & 21.485 & -0.013 \\
\hline-28.5 & 12.713 & 12.715 & -0.002 & -14 & 21.774 & 21.795 & -0.021 \\
\hline-28 & 13.043 & 13.032 & 0.011 & -12.5 & 22.690 & 22.723 & -0.033 \\
\hline-27.5 & 13.362 & 13.348 & 0.014 & 12 & 37.558 & 37.571 & -0.013 \\
\hline-27 & 13.669 & 13.663 & 0.006 & 13 & 38.168 & 38.164 & 0.004 \\
\hline-26.5 & 13.982 & 13.979 & 0.003 & 13.5 & 38.471 & 38.460 & 0.011 \\
\hline-26 & 14.305 & 14.294 & 0.011 & 14 & 38.764 & 38.756 & 0.008 \\
\hline-25.5 & 14.612 & 14.609 & 0.003 & 14.5 & 39.057 & 39.051 & 0.006 \\
\hline-25 & 14.917 & 14.924 & -0.007 & 15 & 39.341 & 39.346 & -0.005 \\
\hline-24.5 & 15.229 & 15.239 & -0.010 & 15.5 & 39.628 & 39.641 & -0.013 \\
\hline-24 & 15.556 & 15.553 & 0.003 & 16 & 39.912 & 39.936 & -0.024 \\
\hline-23.5 & 15.866 & 15.867 & -0.001 & 16.5 & 40.206 & 40.230 & -0.024 \\
\hline-23 & 16.186 & 16.181 & 0.005 & 17 & 40.507 & 40.524 & -0.017 \\
\hline-22.5 & 16.517 & 16.495 & 0.022 & 17.5 & 40.804 & 40.818 & -0.014 \\
\hline-22 & 16.834 & 16.809 & 0.025 & 18 & 41.117 & 41.111 & 0.006 \\
\hline-21.5 & 17.141 & 17.122 & 0.019 & 18.5 & 41.422 & 41.404 & 0.018 \\
\hline-21 & 17.451 & 17.435 & 0.016 & 19 & 41.713 & 41.697 & 0.016 \\
\hline-20.5 & 17.768 & 17.748 & 0.020 & 19.5 & 42.009 & 41.990 & 0.019 \\
\hline-20 & 18.068 & 18.061 & 0.007 & 20 & 42.305 & 42.282 & 0.023 \\
\hline-19.5 & 18.367 & 18.373 & -0.006 & 26.5 & 46.069 & 46.056 & 0.013 \\
\hline-19 & 18.675 & 18.685 & -0.010 & 27 & 46.361 & 46.344 & 0.017 \\
\hline-18.5 & 18.966 & 18.997 & -0.031 & 29 & 47.482 & 47.494 & -0.012 \\
\hline-18 & 19.295 & 19.309 & -0.014 & 29.5 & 47.785 & 47.781 & 0.004 \\
\hline-17.5 & 19.600 & 19.621 & -0.021 & 30 & 48.086 & 48.067 & 0.019 \\
\hline-17 & 19.948 & 19.932 & 0.016 & 31.5 & 48.906 & 48.924 & -0.018 \\
\hline-16.5 & 20.259 & 20.243 & 0.016 & 32 & 49.198 & 49.210 & -0.012 \\
\hline-16 & 20.577 & 20.554 & 0.023 & 32.5 & 49.484 & 49.495 & -0.011 \\
\hline
\end{tabular}
the limits of the experimental errors. The difference

Table 1. Observed and calculated wavenumbers of the fine structure components of the NH stretching band of pyrrole. 
between $B^{\prime \prime}$ and $B^{\prime}$ is small, but it has been proved to be positive by a more appropriate graphical analysis.

Except for the center of the $Q$ branch, the recorded absorption band agrees well with the computed one. The slight deviation from the exact symmetric top causes the greatest discrepancies when $K$ is

- M. C. Allen and P. C. Cross, Molecular Vib-Rotors, John Wiley \& Sons Inc., New York 1963, p. 178. small, because it is there that the strongest components of the parallel type band exist, and these transitions are allowed for in an asymmetric top molecule ${ }^{5,6}$.

The author wishes to thank Dr. R. ANTTILA for great help in recording the spectra and Prof. P. TuoMIKOSKI for suggesting the topic. This work was financially supported by Neste Oy and the National Research Council for Sciences which are gratefully acknowledged.

\title{
Radiation-Induced Conformation Changes of the Pyrimidine Ring in a Single Crystal of Dihydrothymine
}

\author{
J. N. HERAK \\ Institute "Rudjer Bošković”, Zagreb, Croatia, Yugoslavia \\ (Z. Naturforsch. 25 a, 1299—1302 [1970] ; received 10 January 1970)
}

\begin{abstract}
Radiation-induced radicals in a single crystal of dihydrothymine were studied with EPR. It was found that the 5-thymyl radical is the dominant radical species at room temperature. The orientation dependence of the hyperfine couplings can only by explained if one assumes that $\mathrm{C}_{(5)}, \mathrm{C}_{(6)}$ and $\mathrm{C}_{(7)}$ lie in the plane defined by the rest of the nonhydrogen atoms of the ring. The highly disordered structure of the ring in the undamaged crystal is ordered if a proton bound to $\mathrm{C}_{(5)}$ is removed.
\end{abstract}

Radiation damage of several dihydropyrimidines in powder and solution has been studied with electron paramagnetic resonance $(\mathrm{EPR})^{1}$. No similar investigation in single crystals of any of these compounds has been done. In the present paper we report an EPR study of the structure of free radicals in a gamma-irradiated single crystal of dihydrothymine. The crystal and molecular structure of this compound has been reported by FURBERG and JEN$\mathrm{SEN}^{2}$. In contrast to thymine ${ }^{3,4}$, the pyrimidine ring in the crystal of dihydrothymine is highly disordered. From the EPR study of the radiation induced radicals in a single crystal of dihydrothymine it is possible to determine whether or not the disordered structure of the ring remains unchanged after the molecule has been damaged by irradiation.

\section{Experimental}

The crystals were grown by slow evaporation of an aqueous solution at $20^{\circ} \mathrm{C}$. No good quality crystals of moderate size could be found. Fortunately, dihydrothymine is very sensitive to irradiation and the crystal $1 \times 1 \times 0.2 \mathrm{~mm}^{3}$ in size was sufficient for the EPR ana-

1 W. Snipes and W. Bernhard, Radiat. Res. 33, 162 [1968].

2 S. Furberg and L. H. Jensen, J. Amer. Chem. Soc. 90, 470 [1968]. lysis. The external configuration and the crystallographic axes of the crystal used in this study are shown in Fig. 1. The axes were identified with X-ray diffraction analysis. The crystal is orthorhombic and the dimensions of the unit cell are $a=7.336, b=23.474$ and $c=7.034 \AA$ (l. c. ${ }^{2}$ ). There are eight molecules in the unit cell.

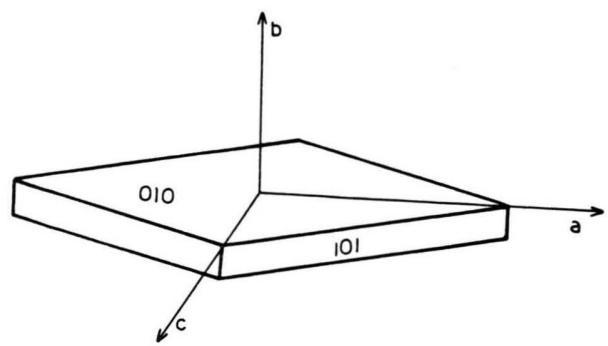

Fig. 1. External configuration and the crystallographic axes of the single crystal of dihydrothymine.

The crystal was irradiated in air at room temperature to a dose of about 5 Mrads by ${ }^{60} \mathrm{Co}$ gamma rays, at a dose rate of $0.3 \mathrm{Mrads} / \mathrm{hour}$. The crystal was aligned in the EPR cavity so that one of the crystallographic axes was perpendicular to the magnetic field. The spectra were recorded at room temperature with a

3 R. Gerdil, Acta Cryst. 14, 333 [1961].

${ }^{4}$ K. Ozeki, N. Sakabe, and J. Tanaka, Acta Cryst. B 25, 1038 [1969]. 